\title{
Evolution and Prospects for Intracranial Pharmacotherapy for Refractory Epilepsies: The Subdural Hybrid Neuroprosthesis
}

\author{
Nandor Ludvig, Geza Medveczky, Jacqueline A. French, Chad Carlson, \\ Orrin Devinsky, and Ruben I. Kuzniecky
} Comprehensive Epilepsy Center, New York University School of Medicine, NYU Langone Medical Center, 223 East 34th Street,
New York, NY 10016, USA

Correspondence should be addressed to Nandor Ludvig, nandor.ludvig@nyumc.org

Received 11 September 2009; Accepted 5 November 2009

Academic Editor: Annamaria Vezzani

Copyright (๑) 2010 Nandor Ludvig et al. This is an open access article distributed under the Creative Commons Attribution License, which permits unrestricted use, distribution, and reproduction in any medium, provided the original work is properly cited.

\begin{abstract}
Intracranial pharmacotherapy is a novel strategy to treat drug refractory, localization-related epilepsies not amenable to resective surgery. The common feature of the method is the use of some type of antiepileptic drug (AED) delivery device placed inside the cranium to prevent or stop focal seizures. This distinguishes it from other nonconventional methods, such as intrathecal pharmacotherapy, electrical neurostimulation, gene therapy, cell transplantation, and local cooling. AED-delivery systems comprise drug releasing polymers and neuroprosthetic devices that can deliver AEDs into the brain via intraparenchymal, ventricular, or transmeningeal routes. One such device is the subdural Hybrid Neuroprosthesis (HNP), designed to deliver AEDs, such as muscimol, into the subdural/subarachnoid space overlaying neocortical epileptogenic zones, with electrophysiological feedback from the treated tissue. The idea of intracranial pharmacotherapy and HNP treatment for epilepsy originated from multiple sources, including the advent of implanted medical devices, safety data for intracranial electrodes and catheters, evidence for the seizure-controlling efficacy of intracerebral AEDs, and further understanding of the pathophysiology of focal epilepsy. Successful introduction of intracranial pharmacotherapy into clinical practice depends on how the intertwined scientific, engineering, clinical, neurosurgical and regulatory challenges will be met to produce an effective and commercially viable device.
\end{abstract}

\section{Introduction}

In the last 20 years, several research groups have explored treating conventionally untreatable epilepsies with delivery of AEDs directly into epileptogenic tissue, the cortical subarachnoid space, or the cerebral ventricles [1-26]. This emerging strategy of "intracranial pharmacotherapy" uses some type of drug delivery device placed inside the cranium. This distinguishes it from both systemic pharmacotherapy, which delivers drugs into the brain through the gastrointestinal, dermal, and/or cardiovascular systems, and from intrathecal pharmacotherapy, which delivers drugs through the theca of the spinal cord. The present article reviews the diverse origins, present state, main challenges, and future prospects for intracranial pharmacotherapy. We focus on our efforts to develop a feedback-controlled intracranial drug delivery device, the subdural HNP, for neocortical epilepsies.

\section{Intracranial Pharmacotherapy in the Context of Epilepsy Treatment Strategies}

Historically, four major strategies have been used for the treatment of epilepsies. These are dietary and behavioral therapy, systemic pharmacology, and neurosurgery. Dietary therapy and behavioral therapy have been practiced for centuries. While effective in many patients, the overwhelming majority of drug refractory epilepsies (DRE) cannot be controlled with these strategies. Neurosurgical interventions reduce or eliminate seizures by either removing the epileptogenic zone (e.g., temporal lobectomy, hemispherectomy, neocortical tissue resection) or destroying the neural pathways of seizure propagation (e.g., by callosotomy, subpial resection). While these strategies can improve or cure many patients, both interventions are burdened with the risk of damaging normal neural tissue. Systemic pharmacology controls seizures in up to $70 \%$ of all patients. However, 
during systemic AED intake the entire body is exposed to the compound, although the targeted epileptogenic zones occupy less than a thousandth of the body mass. Indeed, a neocortical seizure focus with an average tissue volume of $7 \mathrm{~cm}^{3}$ is $10,000 \times$ less than the approx. $70,000 \mathrm{~cm}^{3}$ body volume of a $70 \mathrm{~kg}$ and $180 \mathrm{~cm}$ high person [27]. The Vagus Nerve Stimulator (VNS) of Cyberonics (Houston, TX), approved by FDA for DRE in 1997, marked a new approach to epilepsy therapy. This device belongs to the family of "neuroprostheses", which also includes the Deep Brain Stimulator (DBS) $[28,29]$ and the Responsive Neurostimulation (RNS) system by NeuroPace (Mountain View, CA) [3032]. Intracranial pharmacotherapy is the product of the same intellectual wave that, departing from the conventional therapies, produced the VNS and other brain stimulation devices, as well as the ideas that intracerebral gene transfer $[33,34]$, cell transplantation [35, 36], or local cooling [37] might also be used to treat focal epilepsies.

\section{The Need for Developing Intracranial Drug Therapy for Epilepsy}

Approximately $30 \%$ of the epilepsy patient population will not achieve complete remission of seizures with standard AED therapy [38, 39]. This translates to about 600,000 people in our country and almost 15 million in the rest of the world with DRE $[8,40,41]$. Nearly a third of these patients suffer the severe condition of one or more seizures per month. Many DRE patients, especially those with mesial temporal lobe epilepsy (MTLE), are candidates for neurosurgical intervention. However, about $90 \%$ of patients with severe DRE are unsuitable for surgical tissue resection/lesion [42, 43], because the seizure-generating regions (a) overlap primary sensory, primary motor, or language (Figure 1) areas, (b) occupy too large a tissue mass in one lobe or involve multiple foci which are multilobar and/or bihemispheric or (c) are nonlesional and difficult or impossible to localize. These challenges underline the need to explore the usefulness of intracranial AED delivery. We estimate that there are about 140,000 DRE patients in the US who might be considered as potential candidates for some form of nontraditional epilepsy treatment, including intracranial pharmacotherapy.

\section{Conceptual Evolution of Intracranial AED Delivery}

The idea of treating epileptic seizures with drugs delivered directly into the brain is related to the paradigm shift in medicine that took place in the 1950s and 1960s, leading to the cardiac pacemaker, cochlear implant, and other implanted devices. Microelectronics set the stage for this paradigm shift. Thus, the pacemaker successfully implanted in the initial groups of patients [44] could not be designed without the commercial availability of the transistor; the VNS [45] could not be constructed without the microprocessor.
The neurostimulators and other "neuroprostheses" designed to correct abnormal brain functions [45-47] opened the eyes of the medical community to new possibilities in the treatment of neurological disorders. It has also become clear that with proper neurosurgical techniques and post-implantation care these devices cause no major damage in neural tissue, or at least such damage is not inherent to their use and does not carry substantially more risk than short-term intracranial electrode or catheter placement. The histopathology of brains of Parkinson's disease patients treated with DBS showed "no differences in stimulated and nonstimulated tissues adjacent to the lead-track" [48]. In epilepsy clinical trials, no major side-effects were reported during the course of centromedian thalamic stimulation [28], just as "no adverse stimulation-induced side effects" were observed in epilepsy patients implanted with the RNS device [32]. Thus, while these data obviously could not provide information on whether long-term intracranial drug applications would also be free of side-effects, they suggest that such interventions are not accompanied with prohibitive risks.

Neuropharmacological studies have shown that localized, intracerebral drug applications can modulate, prevent or stop epileptiform EEG and behavioral events [13]. As early as 1970, Collins [49] reported that muscimol, applied topically on the neocortical surface, blocked focal seizures induced by penicillin, bicuculline and picrotoxin, in rats. Muscimol could also suppress audiogenic seizures if injected into the inferior colliculus [50], a structure later proven to be the generator site of sound-induced EEG seizures [51]. Piredda and Gale [52] showed in rats that local application of muscimol into the deep prepiriform cortex can temporarily eliminate epileptogenicity in this region, concluding that this area "may also represent a site at which GABA agonists could function therapeutically to control epileptogenesis". In Smith et al.'s paper [1] describing the antiepileptic effect of lidocaine injected into the deep prepiriform cortex, they suggest that "... microchip and implantable pump technology should make it possible to construct a system that would predict onset of a seizure and then inactivate the neurons in the focus before they could initiate an ictal event". Shortly after, Eder et al. [2] reported that cortically delivered diazepam can attenuate bicuculline-induced local epileptiform EEG spikes, again suggesting the "possible role for AED perfusion directly on seizure focus as a therapy for intractable partial seizures". The seeds for a new therapy for intractable focal epilepsy were sown.

Better understanding of focal epilepsy also contributed. Seizures usually originate in discrete epileptogenic zones (Figures 1(a) and 1(b)), while the rest of the brain may function normally until the electrophysiological seizure activity propagates to neighboring or even more distant structures. This has justified the search for ways to pharmacologically control cortical or subcortical epileptogenic zones, without the unnecessary and often harmful exposure of the body and the rest of the brain to drugs.

Advances in medical device manufacturing, neurostimulation research, intracerebral AED pharmacology and clinical 


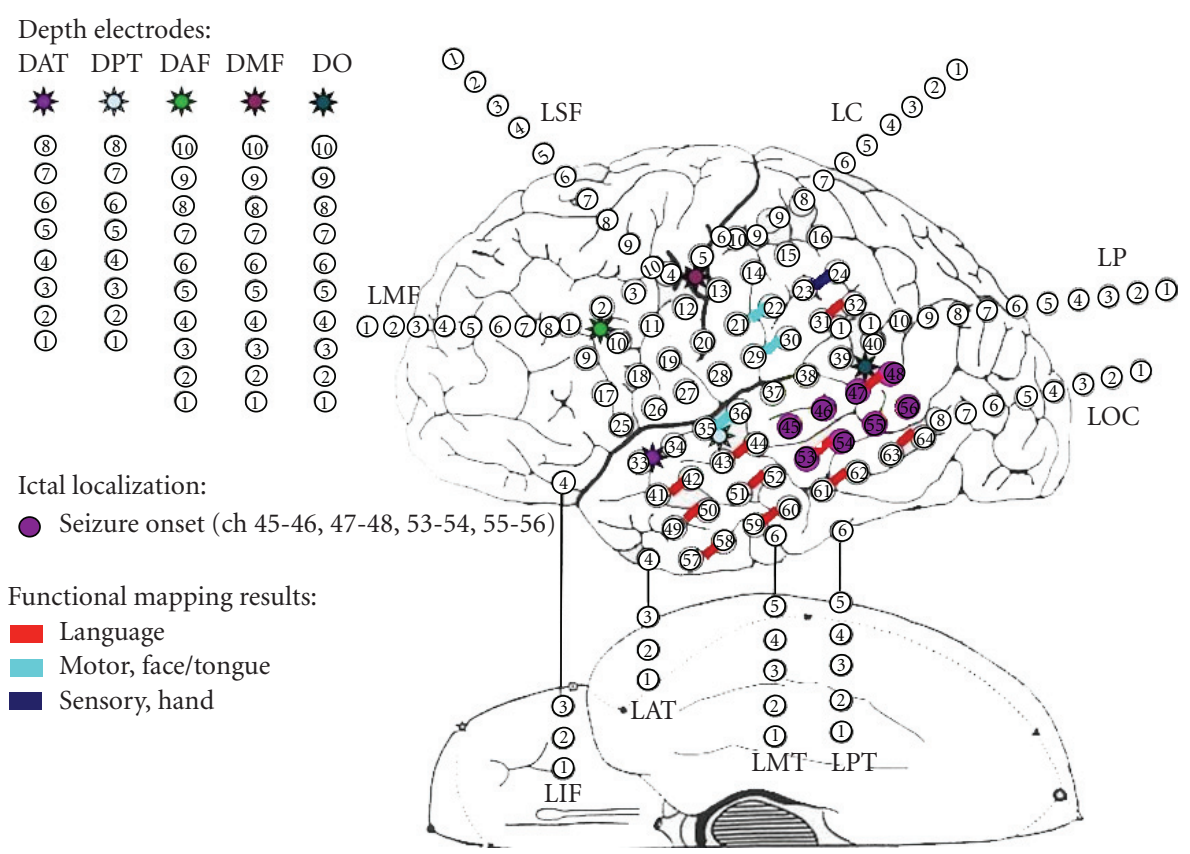

(a)

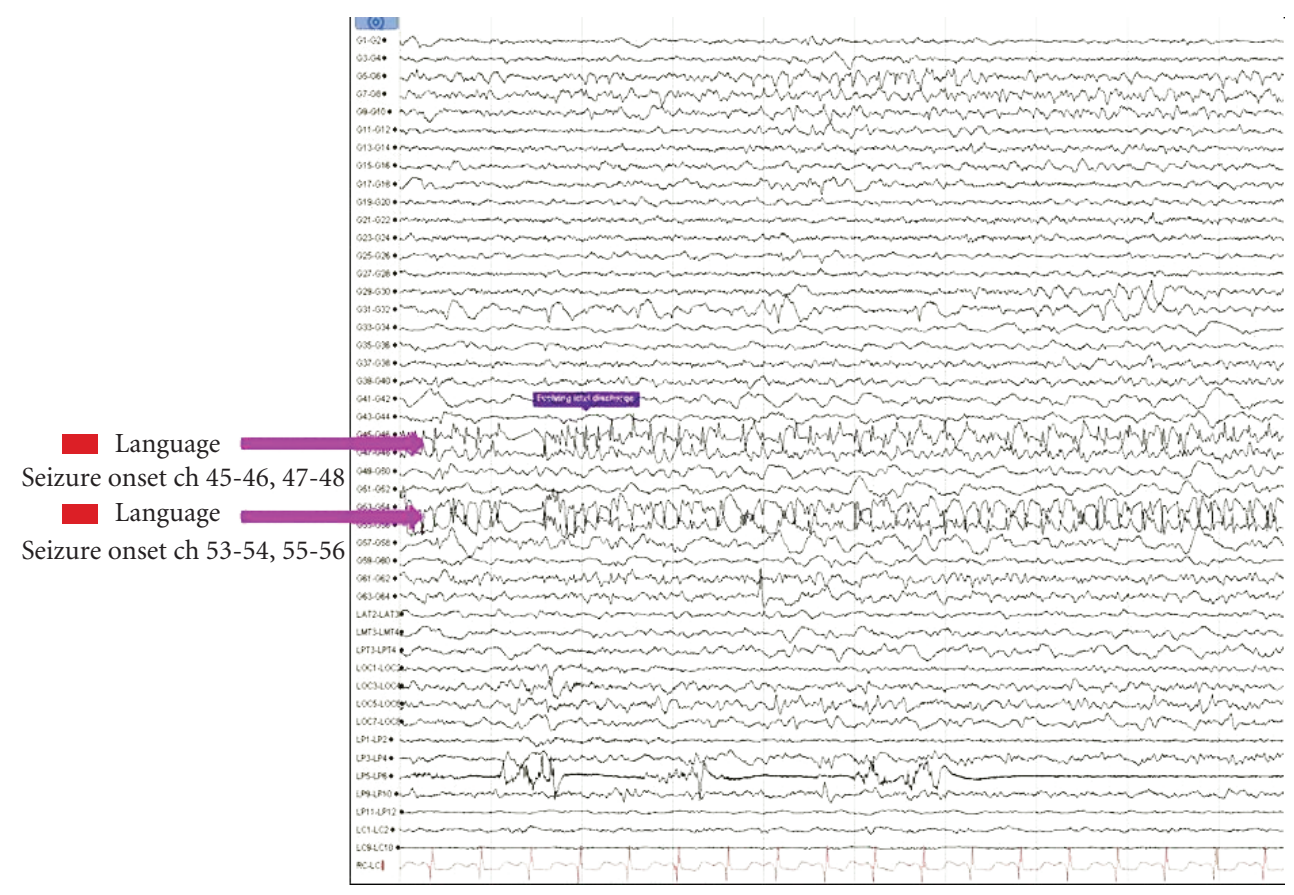

(b)

FIGURE 1: An example of severe focal neocortical epilepsy not amenable to complete tissue resection. (a) Schematic representation of the intracranial electrode array implanted for localization of the ictal onset zone. The ictal onset is shown by magenta-colored electrode circles. Eloquent and motor/sensory cortices, determined by functional mapping, are shown with colored bars between electrode pairs; red: language area, cyan: motor area; blue: sensory area. The overlap of the ictal onset zone with language areas excludes the option of full resection of the epileptogenic tissue. (b) Focal ictal discharge in the left posterior temporal region highlighted by magenta arrows: note overlap with language areas. 
epileptology were synthesized into specific engineering solutions for intracranial pharmacotherapy for focal epilepsy at the beginning of this decade. In 2000, a "microcomputercontrolled intracerebrally implanted drug delivery device, in which the timing and duration of drug deliveries are determined by the implanted brain tissue's own electrical activity" was described [3]. Also in 2000, Stein et al. [6] published a study demonstrating the efficacy of "an automated drug delivery system for focal epilepsy" in rats, concluding that "such therapy might avoid some of the problems inherent to systemic administration of antiepileptic drugs". In 2000 Fischell et al. [5] patented a "responsive implantable system for the treatment of neurological disorders" (US Patent \#6,134,474), although these inventors focused on electrical stimulation and "medication released into the cerebrospinal fluid of the human patient" as the therapeutic interventions. The idea of "focal methods of drug delivery tied to EEG activity" was embraced by investigators at the National Institutes of Health [7] and within a few years the development of intracranial pharmacotherapy for epilepsy has become the objective of several research teams in academia, in some cases closely collaborating with startup companies (e.g., Sierra Neuropharmaceuticals, MedGenesis Therapeutix, and others).

The goal of treating focal epilepsy with intracranially delivered drugs is being pursued in diverse pathways. One strategy involves the intracranial implantation of AEDreleasing polymers [53]. Cortically implanted phenytoinethylene-vinyl acetate (EVAc) controlled-release polymers have been demonstrated to reduce seizures in a cobaltinduced model of focal neocortical epilepsy [11]. A second strategy aims to deliver AEDs into the brain using a different approach: by utilizing fully implanted, responsive or nonresponsive, neuroprosthetic devices. These devices employ either intraparenchymal catheters or catheter/electrode units, or cannulas placed in the cerebral ventricles, or sealed, subdural fluid delivery/recording electrode units overlaying the neocortical epileptogenic zone(s). One promising technique for intraparenchymal drug administration into the seizure focus or foci, with or without electrophysiological recording capability, uses convection-enhanced delivery (CED), which seeks to "distribute a therapeutic agent homogeneously throughout clinically significant volumes of brain parenchyma" [26]. The relative safety of this method was shown in nonhuman primates [16], while its efficacy to reduce the severity of amygdala-kindled seizures in rats was demonstrated by Gasior et al. [21], who administered Ntype calcium blocker conotoxins into the amygdala via CED. Intracerebroventricular AED administration is an alternative site for intracranial drug delivery. However, this strategy addresses systemic, but not CNS-related toxicity [25], at least with the devices and drug delivery protocols that have been tested in intracerebroventricular seizure-control studies. The anesthetic side-effect of intracerebroventricular pentobarbital administration in rats [54] is a pentobarbital action that can be eliminated without decreasing its neocortical seizure-preventing potency by administering this compound transmeningeally into the cortex via a sealed device [17]. This transmeningeal route offers another avenue for intracranial AED administration, with the assistance of the subdural/subarachnoid HNP device.

\section{The Subdural HNP for the Treatment of Neocortical Epilepsies}

The subdural HNP is a type of intracranial drug delivery device, which offers drug deliveries directly into epileptogenic brain tissue, via sealed, single or multiple, regularly flushed, subdural/subarachnoid units equipped with recording electrodes/sensors to provide feedback from the exposed neural tissue [17] (Figure 2). The basic concept and architecture of the device have been described [9, 17, $19,55,56]$. Its key distinguishing feature is the integration of both fluid exchange/drug delivery ports and recording electrodes/sensors into a silicone strip or grid that can be placed in the subarachnoid space (Figure 3 ). Thus, it is this subarachnoid space through which the device delivers AEDs or other seizure-preventing therapeutic solutions into the underlying epileptogenic zone(s). Consequently, the subdural HNP achieves pharmacological/therapeutic effects via drug diffusion through the cerebral arachnoid and pia maters, virtually eliminating the risk of damaging normal neocortical tissue.

This "transmeningeal pharmacotherapy" is based on a known, albeit medically underutilized, physico-chemical property of the cerebral leptomeninges; that is, their permeability to water-soluble molecules. This is why neurotransmitters released into the neocortical extracellular space can diffuse into a fluid collection device placed on the pia mater $[57,58]$. The movement of molecules across the leptomeninges is bidirectional. Thus, water-soluble small molecules, like $\mathrm{N}$-methyl-D-aspartate (NMDA) or methylene blue, penetrate through these membranes into the underlying cerebral cortex and stay close to the delivery area [18] (Figure 4): findings consistent with prior autoradiographic studies [59].

Inclusion of recording electrodes (and in the future neurochemical sensors) in the subdural drug delivery unit gives potential for the device to execute three important functions. First, electrophysiological recordings can provide feed-back on the effects of the delivered drugs, so that their delivery parameters can be flexibly adjusted, eliminating the danger of applying too high, neurotoxic doses, while helping to avoid the application of too low, thus inefficient drug concentrations. Second, these recordings can potentially provide information for the treating physician on the neurophysiological impact of the subdural implant, so that adverse reactions, if these occur, can be recognized and treated early. Third, electrophysiological data acquisition may permit seizure prediction and seizure detection, thus allowing subdural drug delivery in a responsive, on-demand fashion, upon the occurrence of pre-seizure or seizure-onset signals. This set of three feed-back functions separates the subdural HNP from AED-releasing polymers, gene therapy and cell transplantation.

Multiple drug delivery/recording units, shaped either as strips or grids, can obviously also be used. Thus, the device 


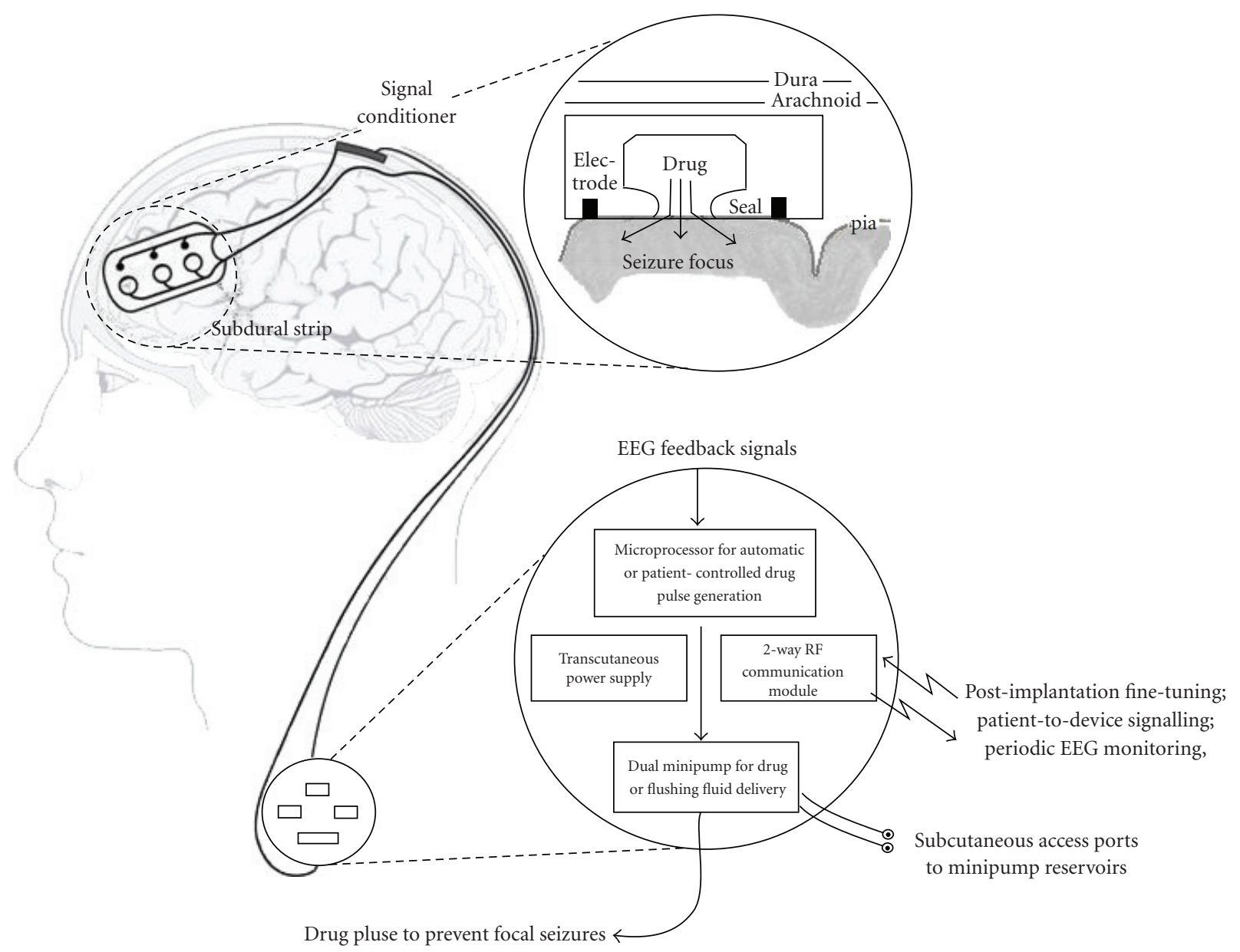

FIGURE 2: Design of the simplest version of the subdural HNP implant, a nonresponsive intracranial pharmacotherapy device. EEG recording from the treated epileptogenic area provides feedback for the electrophysiological effects of drug pulses during post-implantation device checks, so that ineffective delivery parameters, just as potentially hazardous delivery conditions, can be recognized and corrected. This device might be appropriate for a subclass of patients with drug refractory, surgically untreatable neocortical epilepsy, whose frequently occurring or subjectively predictable seizures may not necessitate the use of a responsive apparatus with seizure-prediction/detection capability. Namely, in these patients automatic, intermittent drug pulses or patient-activated drug deliveries with the above device may provide adequate seizure control without side effects. Since the minipump reservoirs can be refilled via subcutaneous access ports, in each patient more than one AED can be tried and used. Should this strategy prove to be successful in clinical trials, it may well pave the way for the next, responsive version of this device [19].

could apply treatment over large cortical areas, without the spatial limitations imposed upon devices using tissuepenetrating cannulas, catheters or tubes. This may allow the treatment of extended, multiple, and/or bilateral seizure foci, as well as diffusely distributed epileptogenic zones that are difficult to localize even with intracranial recordings. Presently, muscimol is emerging as the choice of AED for the first generation subdural HNPs [19, 20], because of the following reasons: (a) cortical seizure-preventing efficacy in low ( $\leq 1 \mathrm{mM}$; Figure 5) concentrations, (b) fast-developing ( $\sim 30$ seconds) pharmacological action, (c) high watersolubility, (d) long-term ( $\sim 4$ month) stability in solution, and (e) efficacy at neutral $\mathrm{pH}$. Another feature of the subdural HNP design is the sealing membrane around both the individual drug delivery ports (Figures 2 and 3 ) and the entire subdural unit. This prevents significant drug spillover to neighboring, normal cortical areas and limits systemic exposure to the administered AED. During pentobarbital administration into the neocortex through a sealed epidural cup (as in rodents the thin, permeable dura mater allows transmeningeal drug application via such devices) focal seizures can be readily prevented, while the rat remains awake [17]. The lack of significant drug spillover into the CSF or the rest of the cerebral cortex is also demonstrated in Figure 5(a). It illustrates that if one side of the frontal cortex is pretreated with transmeningeal saline, while the contralateral site is simultaneously pre-treated with muscimol in the same way, subsequent application of Ach into both cortical areas leads to focal seizures in the saline-treated but not the muscimoltreated side. 


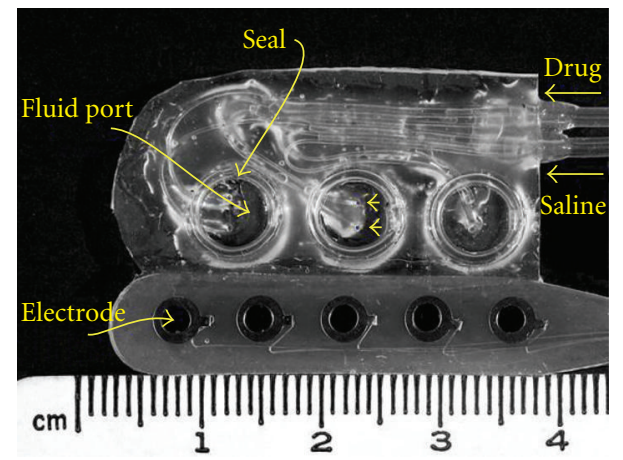

Figure 3: The recording/drug delivery unit of the subdural HNP, designed for tests in nonhuman primates. Photograph shows the side of the unit that faces the pia mater. Two fluid inputs (arrowheads) serve to deliver a drug solution (e.g., muscimol) or a flushing fluid (e.g., saline) into each fluid port. Should the tests in nonhuman primates confirm the safety of this unit, it can be readily adapted to human use. Essentially, this is a combination of a commercially available subdural electrode strip by Ad-Tech (Racine, WI) and a custom-designed fluid-port strip by DocXS Bioemdial Products (Ukiah, CA). No other materials than stainless steel and medical grade silicone are used for its construction. Thickness = $1.2 \mathrm{~mm}$.

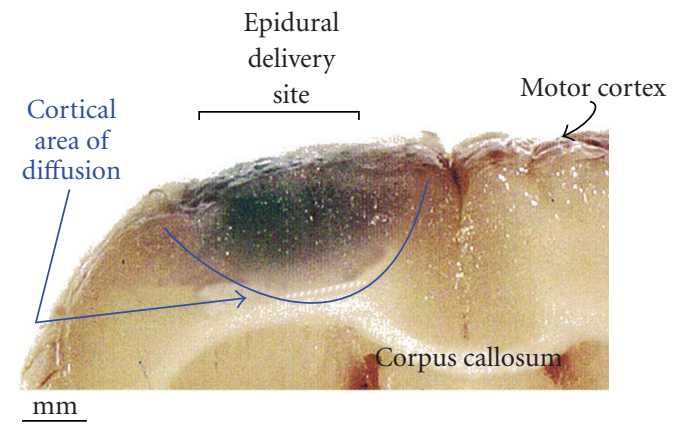

Figure 4: Penetration of methylene blue molecules from the epidural delivery site through the subdural space into the motor cortex, in a rat. Coronal section of a formalin-fixed brain is shown; the concentration of the methylene blue solution was $1 \%$. Note the limited cortical area of diffusion.

The HNP minipump is a dual, fully implantable, and transcutaneously refillable, miniature peristaltic device [20] (Figure 6). Its design and two fluid reservoirs allow the HNP to execute, in an alternating fashion, two functions. One reservoir is for AED delivery, while the other one is for delivering a flushing solution (e.g., saline or artificial CSF) or removing CSF from the subarachnoid space to prevent pressure increase. In the experiment shown in Figure 5(b), muscimol was delivered with this minipump into the cortical subarachnoid space of a freely moving rat: the apparatus was mounted on the head of the animal (Figure 6).

In addition to data generated in rats implanted with epidural drug delivery devices [17-20, 22] (Figures 4-6), monkey and human studies also suggest the therapeutic viability of the subdural HNP. In anthropoid New World

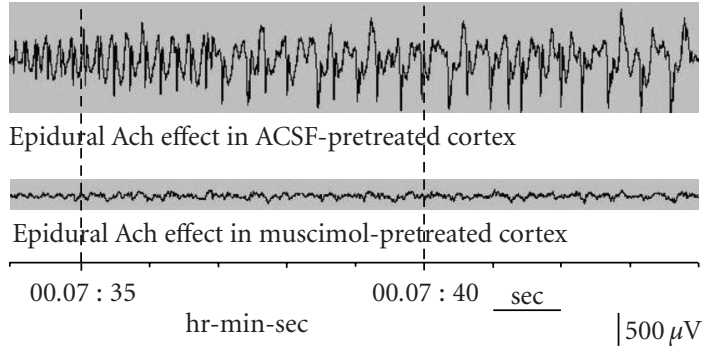

(a)

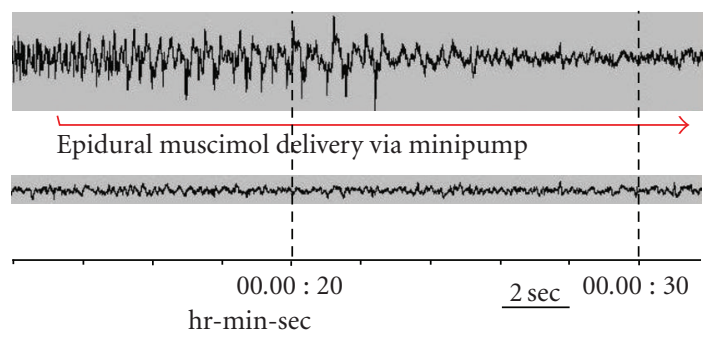

(b)

FIGURE 5: (a): Epidurally administered acetylcholine (Ach) induced focal EEG seizure activity in the left motor cortex pre-treated with artificial cerebrospinal fluid (ACSF), in a freely-moving rat. In contrast, in the same rat simultaneous application of Ach in the right motor cortex pre-treated with $1 \mathrm{mM}$ muscimol could not induce epileptiform EEG activity. (b): In the same experiment, epidural delivery of $1 \mathrm{mM}$ muscimol into the Ach seizure focus stopped the ongoing EEG seizure within 10 seconds. Muscimol delivery was performed with the HNP minipump mounted on the rat's head, an shown in Figure 6.

monkeys (Saimiri sciureus), muscimol delivery into the subarachnoid space prevented focal neocortical seizures [19]. In patients with temporal lobe epilepsy $(n=3)$, in a neurosurgical setting prior to tissue resection, lidocaine (an IRB-approved compound for the HNP project at the time) was applied to the pial surface overlaying the epileptogenic zone. Within minutes, this local treatment markedly reduced the frequency of EEG spiking in the epileptogenic area [24]. Thus, seizure susceptibility in the primate cerebral cortex can be modulated by drugs delivered directly to the pial surface.

\section{Challenges and Prospects}

The prospects of intracranial pharmacotherapy depend on how the intertwined scientific, engineering, clinical, and neurosurgical problems will be solved and the pertinent regulatory and commercial issues navigated. Our initial aim is to investigate the most feasible simplest, nonresponsive version of the subdural HNP in Phase I/II clinical trials, after rat and monkey studies on safety and efficacy are completed. This can set the stage for testing the more complex, responsive HNP version $[9,17,19]$.

The first main scientific challenge of HNP development is the thorough elaboration of the safety profile of the device. Although subdural electrode grids and strips can be routinely kept over the neocortex for a few weeks with minimal or no 


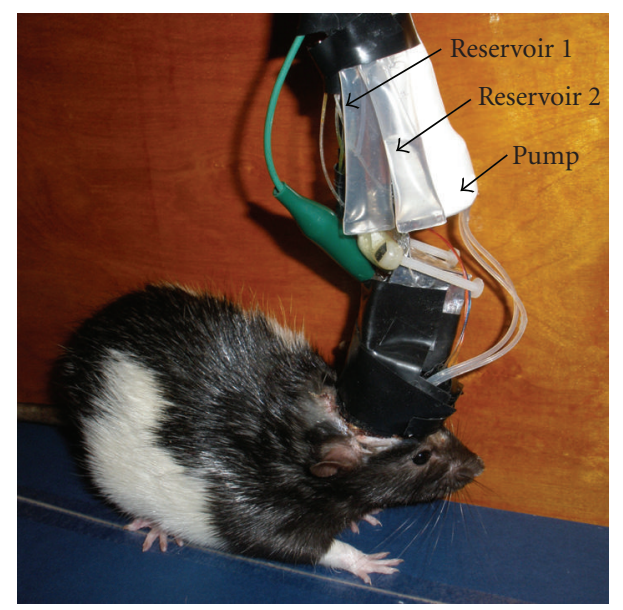

FIGURE 6: Photograph of a rat wearing the dual HNP minipump during an experiment. Depending on the tubing arrangement of the minipump, the device can either direct, in an alternating fashion, two solutions into the brain or can remove CSF from the subarachnoid space before drug delivery. The pump, weighing $30 \mathrm{~g}$, moves fluids to and from two flexible, tissue-compatible silicone reservoirs, of which size can be readily increased to accommodate large volumes for long-term delivery.

complications for diagnostic purposes (Figure 1), the safety of using the subdural unit of the HNP (Figure 3) for months or years to deliver drug solutions must be independently investigated. Second, the potential of tolerance to the applied HNP drugs and the possibility of withdrawal seizures upon the cessation of this treatment must be explored. That tolerance to antiepileptic drugs, including those acting on the GABA system, can develop during their long-term use has been demonstrated by Löscher and Schmidt [60], while the possibility of withdrawal seizures after the cessation of continuous, long-term intracortical GABA administration was shown by Brailowsky et al. [61]. Since muscimol is a GABA-A receptor agonist, clarifying the toleranceinducing and withdrawal-seizure-inducing potency of this drug is essential. The third main scientific challenge is to understand the cellular and neurochemical effects, as well as pharmacokinetics, of transmeningeally delivered AEDs, so that these drugs can be rationally used with intracranial devices. Figure 7 illustrates the complexity of the likely effects and fate of muscimol molecules upon their delivery into the cerebral subdural/ subarachnoid space. Mapping of the diverse cellular actions of this molecule, including its heterogeneous effects on postsynaptic, extrasynaptic and presynaptic GABA-A receptors $[62,63]$, as well as its clearance pattern in the neocortex, require a major research effort. But the furnished information, along with corresponding data for other transmeningeal AEDs, will help to elaborate the optimal delivery conditions for seizure-controlling drugs delivered with the subdural HNP.

The engineering challenges are related to the complexity of the HNP, as it involves both pharmacological and electrophysiological components. This complexity is the product of the goals of (a) delivering drugs into the epileptogenic zones in a feedback-controlled manner, requiring electrophysiological monitoring of the drug-exposed tissue, (b) providing information on the recording and drug delivery functions of the device to the treating physician, with the option of modifying these functional parameters, if needed, requiring the use of a bidirectional RF communication module, and (c) supplying this apparatus with sufficient electrical power for years, requiring the integration of a battery rechargeable transcutaneously via electromagnetic coupling. The drug delivery minipump (Figure 6) consumes about $8 \mathrm{mAh}$ energy, almost 10-times more than the rest of the device, and thus powering of the HNP needs a different engineering solution than what is adequate for the VNS, DBS or RNS. The complexity of the HNP as a seizure-preventing device mimics the evolutionary principle of implementing multiple (neural, endocrine and immune) control systems to maintain physiological functions and prevent disease. But complexity comes with a price, and this price for the subdural HNP is the increased likelihood of hardware errors: a risk less threatening for simpler drug delivery implants. Yet, elimination of this risk is the prerequisite of clinical use.

The clinical challenges include the identification of the ideal candidates for intracranial pharmacotherapy and laying the groundwork for post-implantation patient care. Within the population of focal DRE patients who are not amenable to resective surgery the right subclass for the device needs to be further clarified. Patients with nonlesional focal neocortical epilepsy, "the most challenging group of surgical candidates" [64], are likely candidates for subdural HNP treatment. But this is a heterogeneous group, including patients with frontal, parietal, extramesial temporal and occipital epileptogenic zones, some with involvement of mesial temporal lobe structures, and some with much less easily recognized influences from subcortical structures. The other challenge is to develop infrastructure for postimplantation care and identify protocols for (a) adjusting the right drug delivery parameters (concentration, volume, frequency) for the HNP, based on local recordings transmitted from the implant to the physician, (b) setting up the implant status indicators, and (c) confirming the integrity of the transcutaneous minipump-refilling and battery recharging modules.

The main neurosurgical challenges are to determine (a) the optimal size and shape of the subdural/subarachnoid recording/drug delivery unit (Figure 3), (b) the method for its safe placement over the epileptogenic zones in a way that assures fixed position, (c) the best technique to tunnel the wires and tubing from the HNP controller apparatus to the subdural implant, and (d) the optimal subcutaneous location for the minipump refilling ports and inner coil of the battery recharging circuit.

Whether the subdural HNP, along with other intracranial pharmacotherapy implants, will have a limited niche for the treatment of a quite specific class of patients or it may be useful for a larger patient population even beyond those afflicted by epilepsy, is difficult to predict. Should the first generation of these devices prove to be safe and effective in the initial clinical trials, it will justify the development of the responsive version that could be used in patients with less 


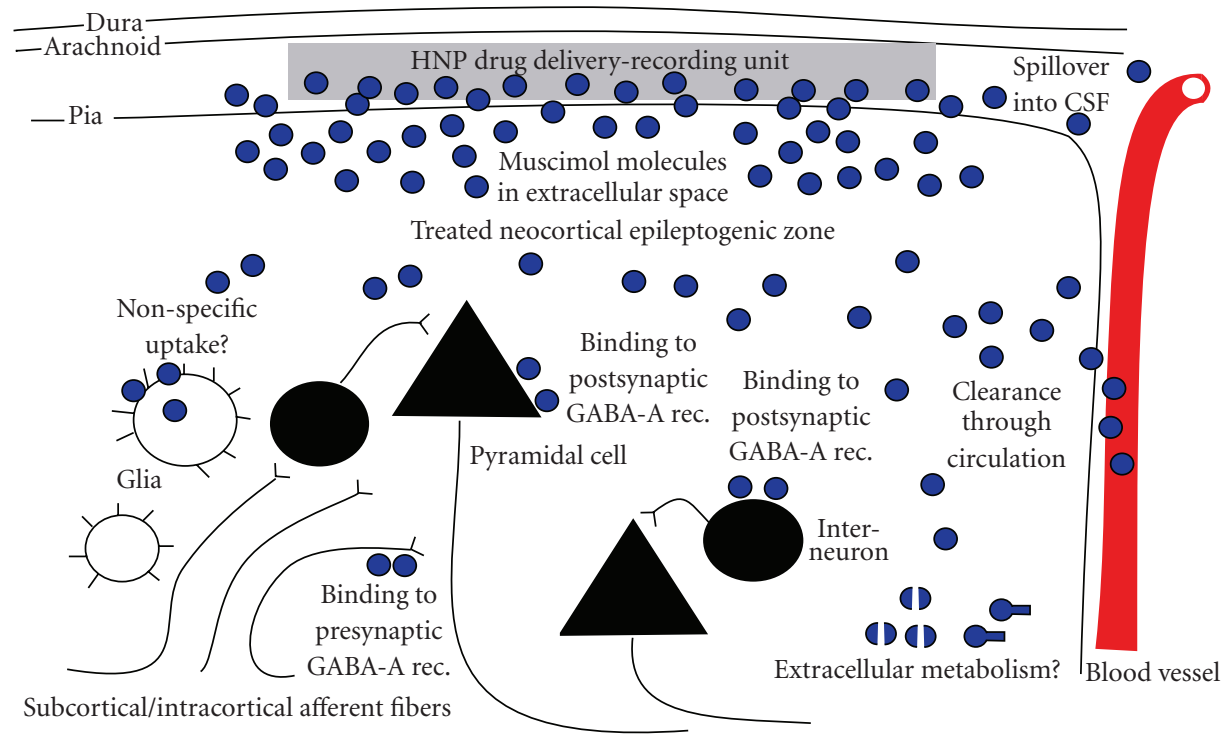

FIGURE 7: Schematic representation of the diverse cellular effects of transmeningeally delivered muscimol in the neocortex and the multiple clearance mechanisms for the cortically diffused molecules. Note that muscimol exerts its primary neuronal effects via GABA-A receptors located postsynaptically on both pyramidal cells and interneurons and presynaptically on both subcortical and intracortical afferent fibers. Besides clearance through cerebrovascular circulation, nonspecific glial uptake and local extracellular metabolism may also contribute to muscimol removal.

frequent seizures. This device may well use microelectrodes to record multi-neuron activity, instead of conventional EEG electrodes, as monitoring cellular electrophysiological signals appears to be more suitable for early seizure detection/prediction and thus for activating AED delivery [65-67]. Newer HNPs may also use a wide spectrum of drugs besides muscimol. Since this device delivers drugs directly into the extracellular space, bypassing the blood-brain barrier (BBB), it can use BBB-impermeable compounds, such as neuroactive peptides and proteins, to achieve therapeutic action. Investigators in other fields of neurology may adapt intracranial pharmacotherapy for maximizing stroke recovery after neocortical infarcts, treating cortical tumors, or improving the cognitive functions in Alzheimer's disease. See Supplementary Material available online at doi:10.1155/2010/725696.

\section{Disclosure}

Orrin Devinsky and Ruben I. Kuzniecky are consultants and members of the Board of Directors at Cortica, Inc., a company founded for the commercialization of the HNP. Geza Medveczky is a consultant for Cortica, Inc.

\section{Acknowledgments}

This work was supported by Finding A Cure for Epilepsy \& Seizures (FACES) and Award 140929 from the Epilepsy Research Foundation. The authors wish to thank the contributions of Dr. Hai M. Tang, Dr. Werner K. Doyle, and Shirn L. Baptiste to the generation of the presented data.
The administrative assistance of Ms. Anjanette N. Burns is greatly appreciated.

\section{References}

[1] D. C. Smith, S. E. Krahl, R. A. Browning, and E. J. Barea, "Rapid cessation of focally induced generalized seizures in rats through microinfusion of lidocaine hydrochloride into the focus," Epilepsia, vol. 34, no. 1, pp. 43-53, 1993.

[2] H. G. Eder, D. B. Jones, and R. S. Fisher, "Local perfusion of diazepam attenuates interictal and ictal events in the bicuculline model of epilepsy in rats," Epilepsia, vol. 38, no. 5, pp. 516-521, 1997.

[3] N. Ludvig, "Drug deliveries into the microenvironment of electrophysiologically monitored neurons in the brain of behaving rats and monkeys," in Neural Prostheses for Restoration of Sensory and Motor Function, J. K. Chapin and K. A. Moxon, Eds., pp. 263-283, CRC Press, New York, NY, USA, 2000.

[4] N. Ludvig and H. M. Tang, "Cellular electrophysiological changes in the hippocampus of freely behaving rats during local microdialysis with epileptogenic concentration of $\mathrm{N}$ methyl-D-aspartate," Brain Research Bulletin, vol. 51, no. 3, pp. 233-240, 2000.

[5] R. E. Fischell, D. R. Fischell, and R. M. Adrian, "Responsive implantable system for the treatment of neurological disorders," US patent no. 6134474, 2000, http://www.uspto.gov/.

[6] A. G. Stein, H. G. Eder, D. E. Blum, A. Drachev, and R. S. Fisher, "An automated drug delivery system for focal epilepsy," Epilepsy Research, vol. 39, no. 2, pp. 103-114, 2000.

[7] M. P. Jacobs, G. D. Fischbach, M. R. Davis, et al., "Future directions for epilepsy research," Neurology, vol. 57, no. 9, pp. 1536-1542, 2001. 
[8] B. Litt, R. Esteller, J. Echauz, et al., "Epileptic seizures may begin hours in advance of clinical onset: a report of five patients," Neuron, vol. 30, no. 1, pp. 51-64, 2001.

[9] N. Ludvig and L. Kovacs, "Hybrid neuroprosthesis for the treatment of brain disorders," US patent no. 6497699, 2002, http://www.uspto.gov/.

[10] R. S. Fisher and J. Ho, "Potential new methods for antiepileptic drug delivery," CNS Drugs, vol. 16, no. 9, pp. 579-593, 2002.

[11] R. J. Tamargo, L. A. Rossell, E. H. Kossoff, B. M. Tyler, M. G. Ewend, and J. J. Aryanpur, "The intracerebral administration of phenytoin using controlled-release polymers reduces experimental seizures in rats," Epilepsy Research, vol. 48, no. 3, pp. 145-155, 2002.

[12] D. J. Anschel, E. L. Ortega, A. C. Kraus, and R. S. Fisher, "Focally injected adenosine prevents seizures in the rat," Experimental Neurology, vol. 190, no. 2, pp. 544-547, 2004.

[13] K. E. Nilsen and H. R. Cock, "Focal treatment for refractory epilepsy: hope for the future?" Brain Research Reviews, vol. 44, no. 2-3, pp. 141-153, 2004.

[14] D. A. Turner, M. A. L. Nicolelis, and K. Landingham, "Preictal seizure detection and demand treatment strategies for epilepsy," in Modern Neurosurgery: Clinical Translation of Neuroscience Advances, D. A. Turner, Ed., pp. 105-118, CRC Press, New York, NY, USA, 2005.

[15] R.-J. Lohman, L. Liu, M. Morris, and T. J. O’Brien, "Validation of a method for localised microinjection of drugs into thalamic subregions in rats for epilepsy pharmacological studies," Journal of Neuroscience Methods, vol. 146, no. 2, pp. 191-197, 2005.

[16] J. D. Heiss, S. Walbridge, P. Morrison, et al., "Local distribution and toxicity of prolonged hippocampal infusion of muscimol," Journal of Neurosurgery, vol. 103, no. 6, pp. 10351045, 2005.

[17] N. Ludvig, R. I. Kuzniecky, S. L. Baptiste, et al., "Epidural pentobarbital delivery can prevent locally induced neocortical seizures in rats: the prospect of transmeningeal pharmacotherapy for intractable focal epilepsy," Epilepsia, vol. 47, no. 11, pp. 1792-1802, 2006.

[18] N. Ludvig, L. G. Sheffield, H. M. Tang, S. L. Baptiste, O. Devinsky, and R. I. Kuzniecky, "Histological evidence for drug diffusion across the cerebral meninges into the underlying neocortex in rats," Brain Research, vol. 1188, no. 1, pp. 228232, 2008.

[19] N. Ludvig, S. L. Baptiste, H. M. Tang, et al., "Localized transmeningeal muscimol prevents neocortical seizures in rats and nonhuman primates: therapeutic implications," Epilepsia, vol. 50, no. 4, pp. 678-693, 2009.

[20] N. Ludvig, H. M. Tang, S. L. Baptiste, et al., "Developing a subdural hybrid neuroprosthesis (HNP) to treat intractable focal epilepsy," in Advances in the Application of Technology to Epilepsy: The CIMIT/NIO Epilepsy Innovation Summit, S. C. Schachter, J. Guttag, S. Schiff, D. C. Schomer, and Summit Contributors, Eds., vol. 16, pp. 3-46, Epilepsy \& Behavior, 2009.

[21] M. Gasior, N. A. White, and M. A. Rogawski, "Prolonged attenuation of amygdala-kindled seizure measures in rats by convection-enhanced delivery of the N-type calcium channel antagonists $\omega$-conotoxin GVIA and $\omega$-conotoxin MVIIA," Journal of Pharmacology and Experimental Therapeutics, vol. 323, no. 2, pp. 458-468, 2007.

[22] J. E. John, S. L. Baptiste, L. G. Sheffield, et al., "Transmeningeal delivery of GABA to control neocortical seizures in rats," Epilepsy Research, vol. 75, no. 1, pp. 10-17, 2007.
[23] W. C. Stacey and B. Litt, "Technology insight: neuroengineering and epilepsy-designing devices for seizure control," Nature Clinical Practice Neurology, vol. 4, no. 4, pp. 190-201, 2008.

[24] D. Madhavan, P. Mirowski, N. Ludvig, et al., "Effects of subdural application of lidocaine in patients with focal epilepsy," Epilepsy Research, vol. 78, no. 2-3, pp. 235-239, 2008.

[25] J. A. Barcia and J. M. Gallego, "Intraventricular and intracerebral delivery of anti-epileptic drugs in the kindling model," Neurotherapeutics, vol. 6, no. 2, pp. 337-343, 2009.

[26] M. A. Rogawski, "Convection-enhanced delivery in the treatment of epilepsy," Neurotherapeutics, vol. 6, no. 2, pp. 344-351, 2009.

[27] J. Sendroy Jr. and H. A. Collison, "Determination of human body volume from height and weight," Journal of Applied Physiology, vol. 21, no. 1, pp. 167-172, 1966.

[28] R. S. Fisher, S. Uematsu, G. L. Krauss, et al., "Placebocontrolled pilot study of centromedian thalamic stimulation in treatment of intractable seizures," Epilepsia, vol. 33, no. 5, pp. 841-851, 1992.

[29] W. H. Theodore and R. Fisher, "Brain stimulation for epilepsy," Acta Neurochirurgica. Supplement, vol. 97, part 2, pp. 261-272, 2007.

[30] K. N. Fountas, J. R. Smith, A. M. Murro, J. Politsky, Y. D. Park, and P. D. Jenkins, "Implantation of a closed-loop stimulation in the management of medically refractory focal epilepsy: a technical note," Stereotactic and Functional Neurosurgery, vol. 83, no. 4, pp. 153-158, 2005.

[31] G. Worrell, R. Wharen, R. Goodman, et al., "Safety and evidence for efficacy of an implantable responsive neurostimulator (RNS) for the treatment of medically intractable partial onset epilepsy in adults," Epilepsia, vol. 46, supplement 8, p. 226, 2005.

[32] W. S. Anderson, E. H. Kossoff, G. K. Bergey, and G. I. Jallo, "Implantation of a responsive neurostimulator device in patients with refractory epilepsy," Neurosurgical Focus, vol. 25, no. 3, article E12, 2008.

[33] F. Noe', J. Nissinen, A. Pitkänen, et al., "Gene therapy in epilepsy: the focus on NPY," Peptides, vol. 28, no. 2, pp. 377383, 2007.

[34] V. Riban, H. L. Fitzsimons, and M. J. During, "Gene therapy in epilepsy," Epilepsia, vol. 50, no. 1, pp. 24-32, 2009.

[35] A. Huber, V. Padrun, N. Déglon, P. Aebischer, H. Möhler, and D. Boison, "Grafts of adenosine-releasing cells suppress seizures in kindling epilepsy," Proceedings of the National Academy of Sciences of the United States of America, vol. 98, no. 13, pp. 7611-7616, 2001.

[36] K. W. Thompson and L. M. Suchomelova, "Transplants of cells engineered to produce GABA suppress spontaneous seizures," Epilepsia, vol. 45, no. 1, pp. 4-12, 2004.

[37] S. Rothman and X.-F. Yang, "Local cooling: a therapy for intractable neocortical epilepsy," Epilepsy Currents, vol. 3, pp. 153-156, 2003.

[38] M. J. Brodie, "Diagnosing and predicting refractory epilepsy," Acta Neurologica Scandinavica, vol. 112, supplement 181, pp. 36-39, 2005.

[39] G. D. Cascino, "When drugs and surgery don't work," Epilepsia, vol. 49, supplement 9, pp. 79-84, 2008.

[40] M. J. Brodie, S. D. Shorvon, R. Canger, et al., "Commission on European affairs: appropriate standards of epilepsy care across Europe," Epilepsia, vol. 38, no. 11, pp. 1245-1250, 1997.

[41] O. Devinsky, "Patients with refractory seizures," The New England Journal of Medicine, vol. 340, no. 20, pp. 1565-1570, 1999. 
[42] B. C. Callaghan, K. Anand, D. Hesdorffer, W. A. Hauser, and J. A. French, "Likelihood of seizure remission in an adult population with refractory epilepsy," Annals of Neurology, vol. 62, no. 4, pp. 382-389, 2007.

[43] H. Choi, G. Heiman, D. Pandis, et al., "Seizure remission and relapse in adults with intractable epilepsy: a cohort study," Epilepsia, vol. 49, no. 8, pp. 1440-1445, 2008.

[44] W. M. Chardack, A. A. Gage, A. J. Federico, G. Schimert, and W. Greatbatch, "Clinical experience with an implantable pacemaker," Annals of the New York Academy of Sciences, vol. 11, pp. 1075-1092, 1964.

[45] R. Terry, W. B. Tarver, and J. Zabara, "An implantable neurocybernetic prosthesis system," Epilepsia, vol. 31, supplement 2, pp. S33-S37, 1990.

[46] J. K. Chapin and M. A. L. Nicolelis, "Brain control of sensorimotor prostheses," in Neural Prostheses for Restoration of Sensory and Motor Function, J. K. Chapin and K. A. Moxon, Eds., pp. 235-261, CRC Press, New York, NY, USA, 2000.

[47] P. R. Kennedy and B. King, "Dynamic interplay of neural signals during the emergence of cursor related cortex in a human implanted with the neurotrophic electrode," in Neural Prostheses for Restoration of Sensory and Motor Function, J. K. Chapin and K. A. Moxon, Eds., pp. 211-233, CRC Press, New York, NY, USA, 2000.

[48] C. Haberler, F. Alesch, P. R. Mazal, et al., "No tissue damage by chronic deep brain stimulation in Parkinson's disease," Annals of Neurology, vol. 48, no. 3, pp. 372-376, 2000.

[49] R. C. Collins, "Anticonvulsant effects of muscimol," Neurology, vol. 30, no. 6, pp. 575-581, 1980.

[50] G. D. Frye, T. J. McCown, and G. R. Breese, "Characterization of susceptibility to audiogenic seizures in ethanol-dependent rats after microinjection of $\gamma$-aminobutyric acid (GABA) agonists into the inferior colliculus, substantia nigra or medial septum," Journal of Pharmacology and Experimental Therapeutics, vol. 227, no. 3, pp. 663-670, 1983.

[51] N. Ludvig and S. L. Moshe, "Different behavioral and electrographic effects of acoustic stimulation and dibutyryl cyclic AMP injection into the inferior colliculus in normal and in genetically epilepsy-prone rats," Epilepsy Research, vol. 3, no. 3, pp. 185-190, 1989.

[52] S. Piredda and K. Gale, "A crucial epileptogenic site in the deep prepiriform cortex," Nature, vol. 317, no. 6038, pp. 623-625, 1985.

[53] M. Kokaia, P. Aebischer, E. Elmer, et al., "Seizure suppression in kindling epilepsy by intracerebral implants of GABA- but not by noradrenaline-releasing polymer matrices," Experimental Brain Research, vol. 100, no. 3, pp. 385-394, 1994.

[54] I. H. Stevenson and M. J. Turnbull, "A study of the factors affecting the sleeping time following intracerebroventricular administration of pentobarbitone sodium: effect of prior administration of centrally active drugs," British Journal of Pharmacology, vol. 50, no. 4, pp. 499-511, 1974.

[55] N. Ludvig, L. Kovacs, R. I. Kuzniecky, et al., "Apparatus and method for monitoring and treatment of brain disorders," US patent application no. 11/224661, 2005.

[56] N. Ludvig, R. Rizzolo, H. M. Tang, R. I. Kuzniecky, and W. K. Doyle, "Microelectrode-equipped subdural therapeutic agent delivery strip," US patent serial no. 61082706, 2008.

[57] J. Y. Wang, T. L. Yaksh, and V. L.W. Go, "In vivo studies on the basal and evoked release of cholecystokinin and vasoactive intestinal polypeptide from cat cerebral cortex and periventricular structures," Brain Research, vol. 280, no. 1, pp. 105-117, 1983.
[58] J. Y. Wang, T. L. Yaksh, G. J. Harty, and V. L. Go, "Neurotransmitter modulation of VIP release from rat cerebral cortex," American Journal of Physiology, vol. 250, pp. R104-R111, 1986.

[59] D. R. Cornblath and J. H. Ferguson, "Distribution of radioactivity from topically applied $\left[\mathrm{H}^{3}\right]$ acetylcholine in relation to seizure," Experimental Neurology, vol. 50, no. 2, pp. 495-504, 1976.

[60] W. Löscher and D. Schmidt, "Experimental and clinical evidence for loss of effect (tolerance) during prolonged treatment with antiepileptic drugs," Epilepsia, vol. 47, no. 8, pp. 1253-1284, 2006.

[61] S. Brailowsky, M. Kunimoto, C. Silva-Barrat, C. Menini, and R. Naquet, "Electroencephalographic study of the GABAwithdrawal syndrome in rats," Epilepsia, vol. 31, no. 4, pp. 369-377, 1990.

[62] T. C. Jacob, S. J. Moss, and R. Jurd, " $G A B A_{A}$ receptor trafficking and its role in the dynamic modulation of neuronal inhibition," Nature Reviews Neuroscience, vol. 9, no. 5, pp. 331-343, 2008.

[63] P. Long, A. Mercer, R. Begum, G. J. Stephens, T. S. Sihra, and J. N. Jovanovic, "Nerve terminal $\mathrm{GABA}_{\mathrm{A}}$ receptors activate $\mathrm{Ca}^{2+} /$ calmodulin-dependent signaling to inhibit voltage-gated $\mathrm{Ca}^{2+}$ influx and glutamate release," The Journal of Biological Chemistry, vol. 284, no. 13, pp. 8726-8737, 2009.

[64] M. S. Duchowny, A. S. Harvey, M. R. Sperling, and P. D. Williamson, "Indications and criteria for surgical interventions," in Epilepsy: A Comprehensive Textbook, J. Engel Jr. and T. A. Pedley, Eds., pp. 1677-1685, Lippincott-Raven, Philadelphia, Pa, USA, 1997.

[65] N. Ludvig, H. M. Tang, S. L. Baptiste, O. Devinsky, and R. I. Kuzniecky, "Neocortical multineuron recording as a potential tool for predicting focal seizures," Epilepsia, vol. 48, supplement 6, p. 388, 2007.

[66] H. Tang, P. Mirowski, S. Baptiste, O. Devinsky, R. I. Kuzniecky, and N. Ludvig, "Evidence for increased neuronal electrophysiological activity before EEG seizure onset in the rat neocortical seizure focus," Epilepsia, vol. 49, supplement 7, p. 382, 2008.

[67] N. S. Artan, P. Mirowski, H. M. Tang, et al., "Detecting abnormally large-amplitude multi-neuron bursts before focal neocortical EEG seizure onset in freely behaving rats," Epilepsia, vol. 50, p. 391, 2009. 


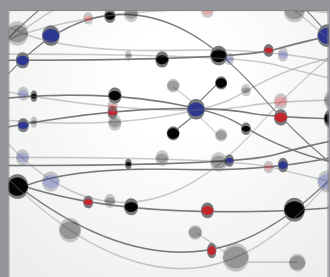

The Scientific World Journal
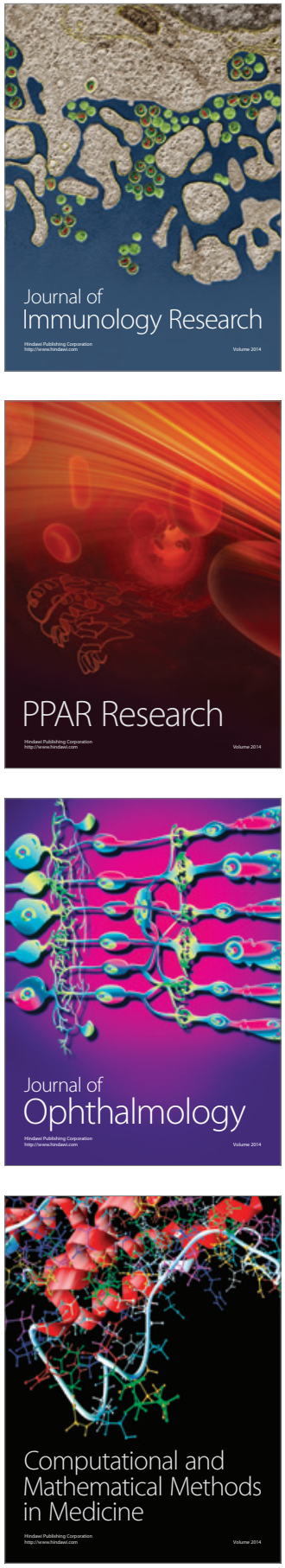

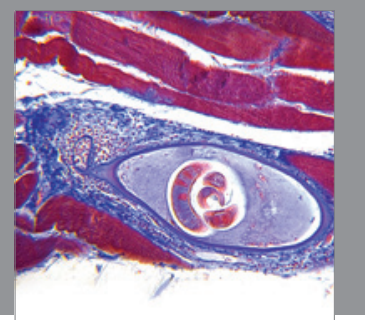

Gastroenterology

Research and Practice
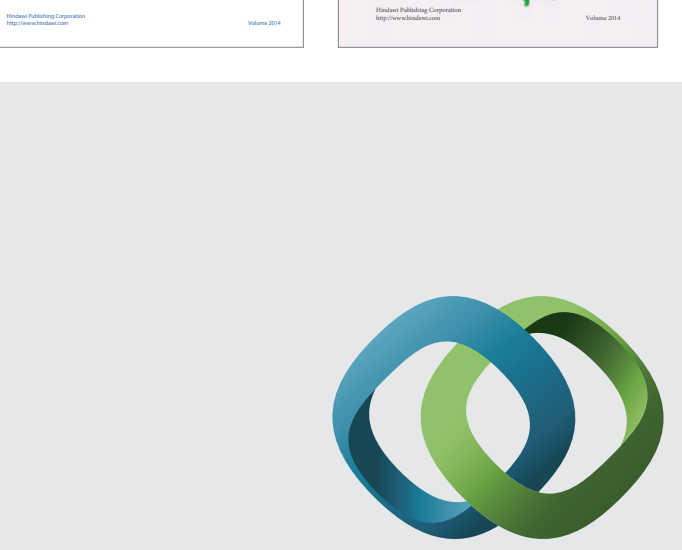

\section{Hindawi}

Submit your manuscripts at

http://www.hindawi.com
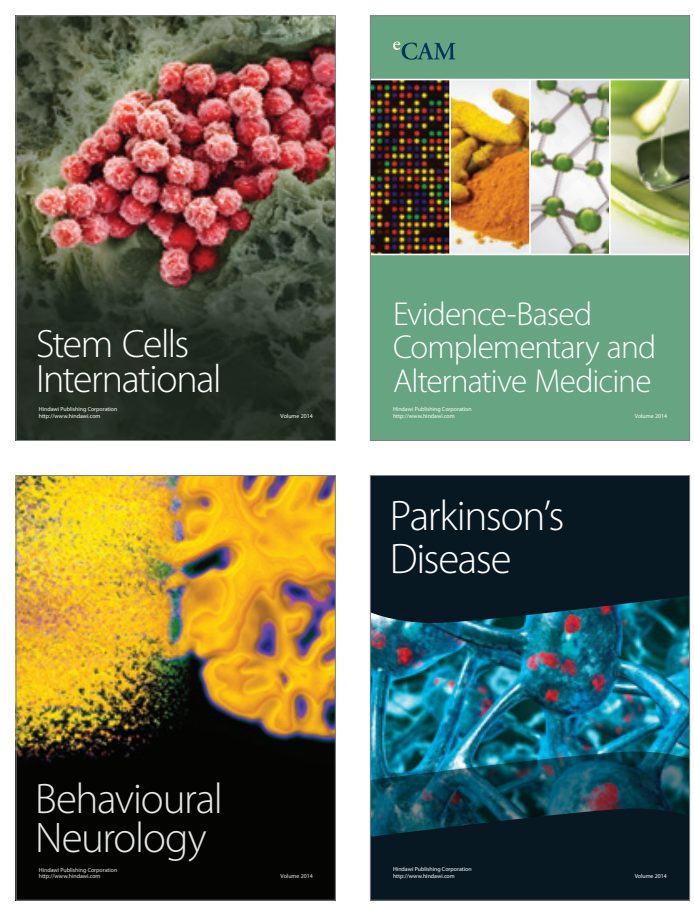

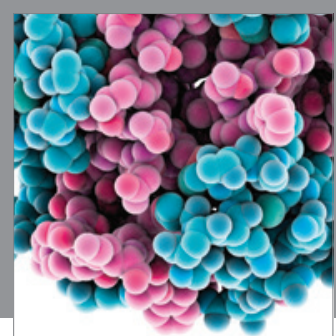

Journal of
Diabetes Research

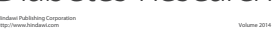

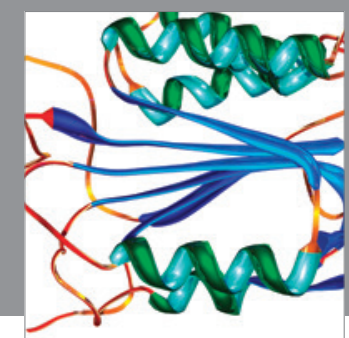

Disease Markers
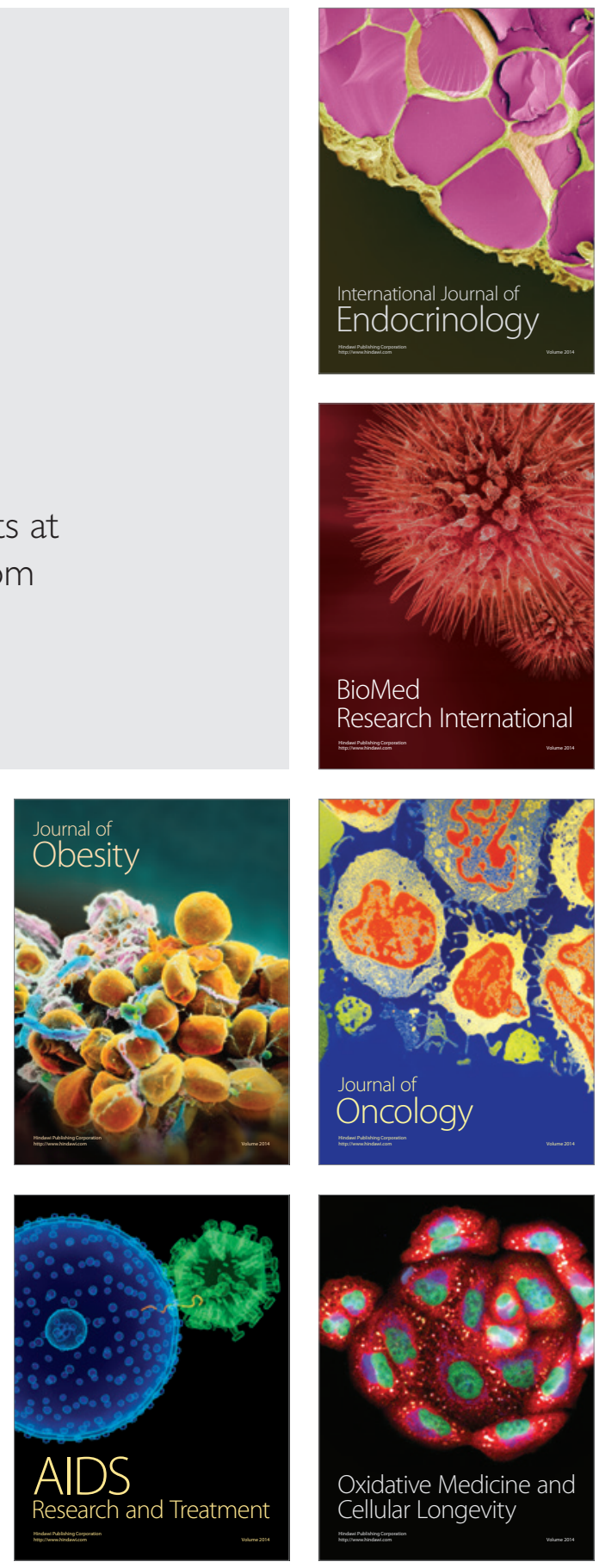